\section{Inositol insulin insight}

\section{By Lev Osherovich, Senior Writer}

A lack of druggable targets in the insulin-Akt pathway has made increasing insulin signaling an uphill struggle for metabolic disease companies. Indeed, there are no approved drugs that directly modulate Akt or other intracellular components of the insulin response. Now, a team from The Johns Hopkins University has found that an inositol-derived signaling molecule called IP7 inhibits Akt as well as glucose utilization, thus opening up a new path for modulating insulin signaling. ${ }^{1}$

Akt (protein kinase B; PKB) is a metabolic regulator that receives signals from the path-

"From a drug discovery
point of view, the data are
very consistent-in the
absence of this enzyme,
you get enhanced insulin
signaling."
-Brian Lavan, Metabolex Inc.

\section{IP address}

Starting with cultured murine embryonic fibroblasts, Snyder's team found that cells lacking IP6K1 had higher Akt activity than wild-type cells. Likewise, a small molecule inhibitor of IP6K1 increased Akt signaling compared with no treatment.

The team went on to characterize the metabolic effects of eliminating IP7 by knocking out IP6K1 in mice. The muscles of IP6K1 knockout mice had higher levels of active Akt and greater glucose uptake than muscles of wild-type controls, suggesting that the absence of IP7 leads to a higher baseline level of insulin signaling.

As insulin signaling helps to package excess glucose into glycogen, Snyder's team predicted that IP6K1 knockouts would be better at controlling blood sugar.

way downstream of the insulin receptor (INSR) to control glucose utilization and protein synthesis.

IP7 (5-diphosphoinositol pentakisphosphate) is a type of inositolpyrophosphate. Inositol pyrophosphates are "a recently appreciated group of messenger molecules about which not much is known," said Solomon Snyder, professor of neuroscience at Johns Hopkins and lead author of a Cell report describing the findings.

Snyder's team previously identified an enzyme, inositol hexakisphosphate kinase-1 (IP6K1), that makes IP7 from a simpler inositol phosphate precursor. ${ }^{2}$ Previous work had shown that IP6K1deficient mice had lower levels of circulating insulin than wild-type mice, prompting the Johns Hopkins group to investigate whether IP7 regulates Akt and insulin signaling.

Figure 1. Inositol phosphates in diabetes. Chakraborty et al. present a case for blocking the formation of 5-diphosphoinositol pentakisphosphate (IP7), a phosphate-rich signaling molecule, to treat obesity, metabolic syndrome and type 2 diabetes.

In the liver, insulin binding to insulin receptors (INSRs) [a] activates a signaling pathway $[\mathbf{b}]$ that ultimately turns on protein kinase $B$ (PKB; Akt) [c], a master regulator of glucose utilization whose activity counteracts the symptoms of metabolic disease [d].

Chakraborty et al. examined the effect of IP7 on metabolism in cell culture and mice and concluded that IP7 inhibits Akt [e], counteracting the effect of insulin.

Because IP7 is produced by the phosphorylation of inositol hexakisphosphate (IP6) by inositol hexakisphosphate kinase-1 (IP6K1), knocking out or inhibiting IP6K1 [f] could increase Akt activity and increase insulin sensitivity, a therapeutic goal in metabolic disease.
Indeed, IP6K1 knockouts had lower blood glucose levels than wild-type animals. Furthermore, the blood sugar of IP6K1 knockouts fed a high-fat diet did not spike as much as that in similarly treated wild-type animals. Overfed IP6K1 knockouts also did not gain as much weight.

Altogether, Snyder's findings suggest that blocking IP7 production by inhibiting IP6K1 increases Akt activity and stimulates glucose utilization (see Figure 1, "Inositol phosphates in diabetes"). Thus, inhibiting IP7 should counteract the effects of a high-fat diet and potentially slow the progression of metabolic syndrome to type 2 diabetes.

The insulin-Akt pathway "has been a target for many therapeutic efforts, but nothing has come of it yet," said Jeff Johnson, principal scientist at Metabolex Inc. Modulating Akt activity by blocking production of IP7 "is another bite at the apple."

Based on the findings, "you would expect that inhibiting IP6K1 would enhance glucose uptake, improving glucose tolerance, and may reduce weight gain or increase weight loss," added Brian Lavan, senior director of biology at Metabolex. "From a drug discovery point of view, the data are very consistent-in the absence of this enzyme, you get enhanced insulin signaling."

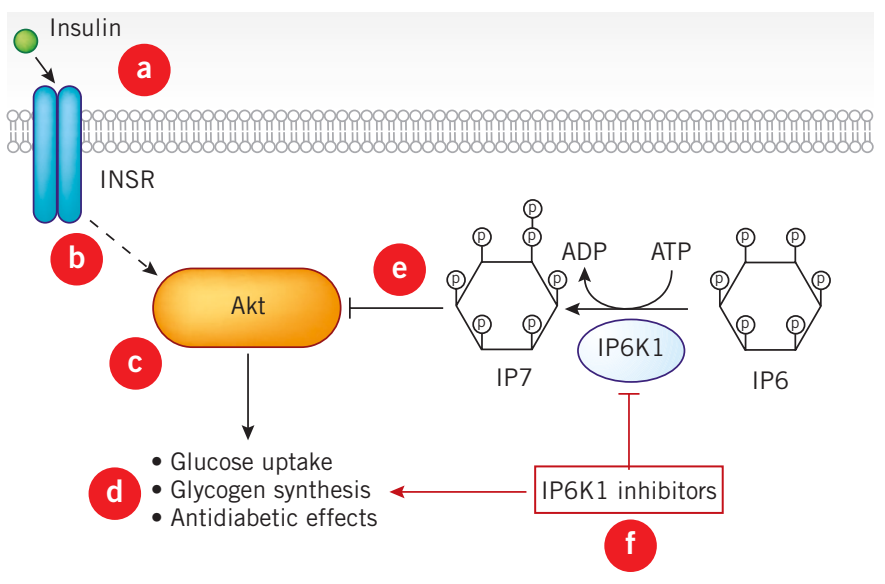




\section{ANALYSIS}

Snyder also said that IP7's effect on Akt appears to be the opposite of that of a better known inositol phosphate-phosphatidylinositol-3,4,5triphosphate (PIP3). PIP3, which is produced in response to insulin binding, activates Akt. He thinks that IP7 competes for the PIP3 binding site on Akt and shuts the enzyme off.

The balance between PIP3 and IP7 "switches cells between activation and inhibition of metabolism," said Snyder.

Lavan noted that previous efforts to alter Akt signaling were aimed at raising PIP3 levels - a much harder proposition because of a lack of good targets. For instance, he said, "people have tried to enhance insulin signaling by targeting phosphatases that either inhibit the insulin receptor" or degrade PIP3.

One such phosphatase is protein tyrosine phosphatase 1B (PTPN1; PTP-1B), which dephosphorylates the insulin receptor. PTP-1B inhibitors in clinical development include TransTech Pharma Inc.'s TTP814 and Ohr Pharmaceutical Inc.'s trodusquemine, which are in Phase II and Phase I testing for type 2 diabetes, respectively.

Both Lavan and Johnson said phosphatases like PTP-1B generally have proven hard to hit, whereas achieving the same metabolic result by inhibiting a kinase like IP6K1 might be more feasible.

Metabolex is tackling type 2 diabetes from a different angle-boosting insulin secretion by pancreatic islet $\beta$ cells. The company's lead compound is MBX-2982, a G protein-coupled receptor 119 (GPR119) agonist that is in Phase II testing for the indication. The compound is partnered with sanofi-aventis Group.

Lavan said that because IP6K1 and GPR119 have such different mechanisms of action, targeting the two proteins concurrently could be complementary.
More work will be needed to discover whether IP6K1 can be selectively inhibited and whether this would have any undesired effects on other metabolic processes.

Snyder said that so far the IP6K1 knockout mice are healthy but have lower than normal levels of insulin due to a homeostatic feedback mechanism. The long-term consequence of low insulin is unknown but should become apparent in follow-up studies.

Meanwhile, Snyder is screening for selective IP6K1 inhibitors. An ideal IP6K1 inhibitor would be able to penetrate into cells and spare related enzymes that are involved in apoptosis, said Snyder. Last year, British academics reported progress toward a selective IP6K1 inhibitor, ${ }^{3}$ but further lead optimization will be needed before preclinical testing can start.

Snyder did not patent his discoveries.

Osherovich, L. SciBX 4(1); doi:10.1038/scibx.2011.1

Published online Jan. 6, 2011

\section{REFERENCES}

1. Chakraborty, A. et al. Cell; published online Dec. 10, 2010; doi:10.1016/j.cell.2010.11.032

Contact: Solomon H. Snyder, The Johns Hopkins University School of Medicine, Baltimore, Md.

e-mail: ssnyder@jhmi.edu

2. Saiardi, A. et al. J. Biol. Chem. 276, 39179-39185 (2001)

3. Padmanabhan, U. et al. J. Biol. Chem. 284, 10571-10582 (2009)

\section{COMPANIES AND INSTITUTIONS MENTIONED}

The Johns Hopkins University, Baltimore, Md.

Metabolex Inc., Hayward, Calif.

Ohr Pharmaceutical Inc. (OTCBB:OHRP), Salt Lake City, Utah sanofi-aventis Group (Euronext:SAN; NYSE:SNY), Paris, France TransTech Pharma Inc., High Point, N.C. 